\title{
REACTIVACIÓN CULTURAL MAPUCHE Y PROCESOS ETNOPOLÍTICOS EN LA CIUDAD. LAS OFICINAS DE ASUNTOS INDÍGENAS EN LA REGIÓN METROPOLITANA, CHILE.
}

\section{Claudio ESPINOZA ARAYA ${ }^{1}$ Rosario CARMONA YOST ${ }^{2}$}

\section{Resumen}

El artículo describe y analiza la relación entre los procesos de reactivación cultural mapuche urbano y la conformación de oficinas de asuntos indígenas en la Región Metropolitana, Chile. Con base en un estudio etnográfico realizado entre 2013 y 2015, proponemos que estas oficinas han introducido la demanda indígena, principalmente mapuche, en la esfera municipal y facilitado la participación de nuevos actores políticos que disputan espacios de poder al interior de la institucionalidad estatal.

Palabras clave: mapuche; indígena urbano; municipio; participación; proceso etnopolítico

\begin{abstract}
This article describes and analyzes the relationship between the processes of urban mapuche cultural reactivation and the constitution of indigenous affairs offices in the Metropolitan Region of Santiago, Chile. Based on ethnographic research conducted between 2013 and 2015, we propose that these offices have introduced in the

\footnotetext{
${ }_{1}^{1}$ Antropólogo. Académico de la Escuela de Antropología de la Universidad Academia de Humanismo Cristiano, investigador asociado del Centro de Estudios Interculturales e Indígenas-CIIR, Proyecto CONICYT/FONDAP/15110006, e investigador responsable del Proyecto Fondecyt de Iniciación 11130002. Condell 506, Providencia, Santiago de Chile. cespinoza@academia.cl

${ }^{2}$ Estudiante de doctorado en Antropología, Universidad de Bonn. Magíster en Antropología por la Universidad Academia de Humanismo Cristiano. Licenciada y magíster en Artes Visuales por la Universidad de Chile. Condell 506, Providencia, Santiago de Chile rosariocarmonayost@gmail.com
} 
municipalities the indigenous request, mainly Mapuche, and facilitated the participation of new political actors who dispute spaces of power among the state institutions.

KeyWords: Mapuche; urban indigenous people; municipality; participation; ethnopolitical process.

\section{Résumé}

Cet article décrit et analyse le lien entre les processus de réactivation culturelle des mapuche urbains et la création de bureaux d'affaires indigènes dans la région Métropolitaine de Santiago du Chili. Cet étude ethnographique a été réalisé entre 2013 et 2015. En conclusion nous considérons que ces bureaux ont permis l'introduction de la demande indigène, principalement mapuche, dans l'espace municipale et qu'ils ont facilité la participation de nouveaux acteurs politiques reivindiquant des places de pouvoir à l'intérieur des institutions d'état.

Mots clés: mapuche; indigènes urbain; municipalité; participation; processus ethno politique.

\section{Introducción}

En este artículo nos proponemos describir y analizar algunos de los procesos que permitieron la configuración de nuevos espacios políticos en los municipios de la Región Metropolitana, particularmente en la ciudad de Santiago, capital de Chile. Se trata de espacios que, en buena medida, son producto del accionar de actores y organizaciones indígenas, principalmente mapuche, que, en conjunto con la política multicultural de los gobiernos pos dictatoriales, han permitido canalizar de manera compleja parte de la participación y la representación de organizaciones indígenas urbanas en los gobiernos locales de la ciudad.

Estos espacios institucionales, denominados oficinas de asuntos indígenas (en adelante OAI), fueron concebidos para atender exclusivamente las demandas de la población indígena. En los últimos veinte años se han multiplicado y transformado en referente para otros municipios del país, que han replicado su modelo en comunas con alta población indígena en regiones del norte y sur de Chile.

Proponemos que la constitución de estos espacios no solo ha permitido el posicionamiento de las demandas de la población indígena urbana en la agenda del municipio, sino también la participación de nuevos actores políticos en los gobiernos locales, materializando con ello eventuales proyectos etnopolíticos y abriendo, aunque limitados, nuevos espacios de poder al interior de la institucionalidad estatal.

El proceso desplegado en la conformación de las OAIs en la Región Metropolitana resulta complejo de analizar. Esto debido a que si bien se han multiplicado progresivamente - 
hoy veintisiete de los cincuenta y dos municipios de la región cuentan con una $\mathrm{OAI}-$, la percepción respecto a estas se ha modificado a la misma velocidad, tanto para los funcionarios que las administran como para el resto de la población indígena urbana, las autoridades municipales e incluso para los distintos gobiernos de los últimos años. Además, estos espacios casi siempre provienen y repercuten en las asociaciones y organizaciones comunales, al vincularse directa o indirectamente con ellas a través de las políticas públicas y los programas sociales dirigidos a la población indígena. Lo anterior implica la administración de los recursos que les son destinados a las organizaciones y, con ello, nuevas alianzas y divisiones.

Por otro lado, desde el año 1996, año en que la Corporación Nacional de Desarrollo Indígena (CONADI) ${ }^{3}$ instala una de sus sedes en Santiago, la temática urbana se ha posicionado progresivamente como tema prioritario de la política indígena. Dentro de este proceso, las OAIs han cobrado protagonismo y adquirido nuevas atribuciones a través del Programa Indígena Urbano (PIU) y otros programas establecidos en alianza con la CONADI y otros ministerios. Debido a esto, el rol y las atribuciones de las OAIs ha aumentado, no obstante, hasta la fecha no se ha diseñado un marco legal específico que las constituya o reglamente.

En este artículo presentamos una aproximación al proceso de conformación y desarrollo de las OAIs de la Región Metropolitana. Para ello comenzamos revisando los procesos migratorios de mediados del siglo XX que afectaron a la sociedad mapuche y que dieron paso a dinámicas de recreación cultural en el contexto urbano. Luego analizamos estas expresiones en relación con los campos de fuerza más amplios que lo envuelven, refiriéndonos específicamente al contexto de transición democrática que vivió Chile a partir de la década de 1990 y a los elementos que lo acompañaron, en especial a las políticas de corte multicultural que promovieron los gobiernos de la transición. Por último, abordaremos un caso paradigmático de las OAIs urbanas, la Oficina de Asuntos Indígenas de la comuna de La Pintana, la primera en constituirse, para concluir después con algunas reflexiones respecto a algunas de las implicancias de este proceso.

Para describir este proceso y desarrollar este análisis nos basamos en los datos obtenidos a través de una investigación cualitativa, principalmente etnográfica, sobre las OAIs, conducida entre los años 2013 y 2015, que tiene como antecedente un estudio realizado por el Núcleo de Estudios Étnicos y Multiculturales de la Escuela de Antropología de la Universidad Academia de Humanismo Cristiano entre los años 2010 y $2011^{4}$. Las OAIs en la Región Metropolitana fueron identificadas a través de consulta directa en los municipios. Los datos cualitativos fueron recogidos a través de observación participante, entrevistas semi-estructuradas y entrevistas en profundidad. Se indagó sobre las motivaciones iniciales, aspiraciones, percepciones, actividades, alcances, limitaciones y proyecciones de cada oficina. Se observaron también las interacciones entre la OAI y la población indígena de la comuna. Estos datos se han complementado con análisis documental y de prensa.

\footnotetext{
${ }^{3}$ La Corporación Nacional de Desarrollo Indígena (CONADI) es la institución estatal responsable de los asuntos indígenas desde 1993 a la actualidad. Dependiente del Ministerio de Desarrollo Social, ha tenido como objetivos la promoción, coordinación y ejecución de las políticas estatales hacia los pueblos indígenas.

${ }^{4}$ En ese proyecto participaron las estudiantes de antropología Pilar Abarzúa, Carolina Altamirano, Rocío Castro, Ximena Cifuentes, Sandy Coloma, Vjera Leyton, Javiera Maham, Fabiola Miranda y Daniela Serey.
} 
Concretamente, las actividades observadas fueron aquellas promovidas por las mismas oficinas, tales como encuentros, reuniones, ferias y celebraciones culturales. Respecto a las entrevistas, estas se realizaron a las encargados y los encargados de las OAIs, así como a funcionarios municipales y usuarios indígenas.

\section{Migración y reactivación cultural en el contexto urbano}

La migración del pueblo mapuche hacia la ciudad de Santiago se produjo desde comienzos del siglo XX, y obedeció a un proceso que tuvo su principal fuente en el progresivo empobrecimiento de las comunidades, el que fue ocasionado a su vez tanto por el menoscabo del territorio como consecuencia de un sistema de tenencia de tierra impuesto desde fines del siglo XIX que conllevó expropiaciones, ventas irregulares y usurpaciones, así como por la reconversión de la economía rural regional (Cuminao y Abarca, 2002). Tal escenario, en inversa proporción con el crecimiento sostenido de la población, provocó que gran cantidad de población mapuche, en su mayoría mujeres jóvenes ${ }^{5}$ (Bello, 2002), decidiera buscar oportunidades laborales en contextos urbanos y fuera de las comunidades.

Debido a que los censos anteriores a la década de 1990 no especifican la adscripción étnica, y los datos que arrojan con respecto a la población mapuche no son confiables (Bengoa, 1996), solo se puede estimar que la migración de la población mapuche hacia las ciudades se intensificó en las décadas de 1950 y 1960 y que llegó a representar el 25\% de la población mapuche total (Bello, 2002). Producto de la promulgación en 1979 del Decreto Ley 2.568, conocido como la Ley de división de las Reservas y de la liquidación de las Comunidades Indígenas, este fenómeno se incrementó y causó que muchos mapuche llegasen a vivir a los sectores periféricos de la ciudad de Santiago, contándose las comunas de Puente Alto, La Pintana y Cerro Navia ${ }^{6}$ entre las principales (Campos, 2002). Por ello es que la Región Metropolitana en general, y la ciudad de Santiago en particular, han acogido a más migrantes mapuche a nivel nacional (Antileo, 2008), contando actualmente con dos y tres generaciones nacidas en la ciudad y con alrededor del $30 \%$ de la población mapuche total (CASEN, 2015).

Como quedó establecido en el informe del grupo de trabajo de Indígenas Urbanos de la Comisión de Verdad Histórica y Nuevo Trato (2008), estos migrantes debieron enfrentarse a una ciudad racista y con falta de viviendas; muchos incluso debieron dormir en sus lugares de trabajo, los que además eran mal remunerados y de gran demanda horaria. Esta situación promovió una serie de tomas de terreno en las comunas periféricas de la ciudad que, si bien al comienzo no consideraron de manera explícita la

\footnotetext{
${ }^{5}$ Ya el Censo de 1992 arrojó una diferencia de género sobre la población residente en la Región Metropolitana, 200.863 hombres mapuche mayores de 14 años y 208.216 mujeres. Esto se explica debido a dos causas: primero, muchas mujeres encontraron trabajo como trabajadoras de casa particular y partían directamente a vivir a las casas que las empleaban, con lo que ahorraban tanto en vivienda como en transporte; segundo, muchas de estas mujeres eran madres, y migraron hacia las ciudades en búsqueda de una mejor educación y oportunidades para sus hijos.

${ }^{6}$ Hoy las comunas que cuentan con mayor población mapuche son Maipú, con 11.913 habitantes, Peñalolén, con 10.369, La Florida, con 11.178, Pudahuel, con 8.480, El Bosque, con 6.069, Puente Alto, con 14.729, y La Pintana, con 11.647 (Censo 2002).
} 
reivindicación étnica (Campos, 2002), gestaron un nuevo sentido de pertenencia y promovieron la asociatividad en torno a la organización barrial y los sindicatos de trabajadores. Debido a que muchos mapuche se insertaron en el mundo laboral de la capital ejerciendo el oficio de panadero, en el caso de los hombres, y de trabajadoras de casas particulares, en el de las mujeres, las agrupaciones ANECAP (Asociación Nacional de Trabajadoras de Casa Particular) y CONAPAN (Confederación Nacional de Panaderos de Chile) resultaron emblemáticas. Ya organizados dentro de estas instancias, muchos indígenas se percataron de lo común de su situación, no solo como trabajadores, sino en tanto que indígenas migrantes. Esta toma de conciencia desencadenó la inclusión de la variable étnica dentro de sus demandas y reorientó la asociatividad en torno a la identidad.

Por otro lado, la migración y la discriminación padecida en la ciudad contribuyeron a que muchos mapuche se desvincularan de sus tradiciones y prácticas realizadas en el sur del país. Esta desvinculación ha sido comprendida como producto de un proceso de asimilación forzada en el que, paulatinamente, la población indígena de las ciudades fue desplazando de su cotidianeidad algunas de las expresiones más visibles de su distinción étnica, transformándose en grupos marginales empobrecidos de características bastante similares a las de otros grupos de pobladores no indígenas. Sin embargo, esta desvinculación no fue absoluta, pues el regreso de los mapuche a sus comunidades de origen fue constante, sobre todo en ocasión de la realización de ceremonias o la celebración de ritos como el we tripantü (solsticio de invierno, denominado como el "año nuevo mapuche") y el nguillatun (rogativa). Este lazo permitió que muchos de ellos se mantuvieran cercanos a las tradiciones, las que más tarde buscarían recrear en la ciudad.

De este modo, revertir la desvinculación con las tradiciones se ha configurado como uno de los primeros y principales objetivos de la población mapuche urbana. Durante los últimos treinta años, cada vez con más firmeza, los mapuche han gestionado la reactivación y visibilización consciente de su cultura, recreando o reinventando tradiciones que se transforman en una herramienta de resistencia cultural y, con ello, política. Al conseguir visibilizar su imaginario en el contexto urbano, los mapuche han sido capaces de explicitar la permanencia de su cultura (Bengoa, 2000; Antileo, 2008; Devalpo, 2008). Una expresión de lo anterior es la construcción de centros ceremoniales y rukas (vivienda mapuche de carácter prehispánico) en la ciudad, en las cuales se realizan diversas actividades, algunas de carácter ancestral mapuche y otras de carácter intercultural, en alianza con diversos programas de gobierno, tales como el Programa Especial de Salud para Pueblos Indígenas (PESPI) y el Programa de Educación Intercultural Bilinguie (PEIB). Por lo tanto, estos espacios se han configurado como lugares donde confluyen el patrimonio material e inmaterial del pueblo mapuche, pero también como lugares que administran la oferta pública y acogen ciertas negociaciones políticas. Es decir, las rukas, y el contexto que las acoge, no se encuentran aisladas, sino que se insertan en las dinámicas urbanas y el escenario supralocal, lo que las recontextualiza constantemente a través de actores que resignifican y reterritorializan el espacio, dotándolo, y a las prácticas que en ellos se desarrollan, de una carga performativa que convoca y genera asociatividad en torno a lo político (Carmona, 2015).

La cultura mapuche en la ciudad, su puesta en valor y acción, se ha posicionado, por lo tanto, como convocante de un grupo bajo el sentido de tradición y pertenencia. Es así como la cultura pasa a ser modificada constantemente por sus actores y el contexto urbano, promoviendo a su vez modificaciones identitarias, o más bien, modificando los 
sistemas de identificación colectivos bajo una misma práctica recontextualizada (Boccara, 2000). Producto de lo anterior, la existencia del sujeto mapuche en la ciudad ha supuesto el surgimiento de un nuevo tipo de memoria histórica para el pueblo mapuche, formulada con arreglo a la nueva coyuntura, la del contexto urbano de carácter modernizador. Los procesos de etnicidad llevados a cabo por actores mapuche en la Región Metropolitana se pueden comprender, por lo tanto, no como la construcción de una nueva identidad en la ciudad, sino como una nueva forma de representarla (Bartolomé, 2006). Representación que amerita ser reflexionada en cuanto persigue y desencadena procesos políticos de mayor alcance.

Bajo esta línea, intelectuales, líderes y/o dirigentes mapuche han señalado que la ética de la acción política se fundamenta en "un proceso de autoafirmación mapuche" (Llaitul y Arrate, 2012) que se construye sobre la base de una tradición cultural que se remonta a tiempos pasados y se concreta a través de la puesta en acción de la identidad, en este caso étnica (Bartolomé, 2006). Para que estas prácticas ligadas a la identidad fueran recodificadas en función de un nuevo posicionamiento que adquiere connotaciones políticas, en donde costumbre y ritual también operan como demarcadores étnicos, se requirió de actores que promovieran y gestionaran su realización. Tales actores se desenvuelven en un contexto urbano que los influencia y con ello, modifica la gestión y la realización de las prácticas rituales y culturales en la ciudad.

Numerosas organizaciones indígenas han construido fuertes discursos en torno a la contextualización de lo urbano desde una postura determinada y con fines altamente políticos (Antileo, 2008), en los que interpelan inclusive a las comunidades y los dirigentes del sur, en el territorio histórico mapuche. La situación de los mapuche urbanos, denominados por algunos intelectuales como warriache (warria: ciudad, che: gente) (Aravena, 2003) o mapurbe (Aniñir, 2009), se ha caracterizado precisamente por la falta de lugar. No solo la falta material producto de la usurpación de los territorios del sur, sino también la falta de un lugar simbólico. Esto, sumado al comportamiento racista y discriminatorio que ha caracterizado la relación de la sociedad nacional con los pueblos originarios, ha promovido la conformación de una corriente indígena urbana fuertemente politizada, que si bien no representa un porcentaje tan amplio en relación con los habitantes en la ciudad, sí ha logrado posicionar sus demandas en busca de mejorar tanto sus condiciones materiales como culturales.

Algunos autores han abordado la conformación de este "sector urbano" del pueblo mapuche. Según Cuminao y Abarca (2002), los enfoques construidos para analizar la migración han sido fundamentalmente dos, por un lado, el que se refiere al aspecto histórico, que comprende la conformación de una población urbana desde los procesos sociales, culturales y económicos que desencadenaron el tránsito del cambio a la ciudad $\mathrm{y}$, por otro, uno que enfatiza los aspectos culturales, en los que la migración en sí misma sería una práctica habitual mapuche. Por otro lado, también hay autores que critican la calificación de urbano porque pareciera implicar una legitimación de la usurpación, instalando esa nueva memoria mapuche en contraposición a la rural, producto precisamente de la dicotomía rural/urbano impuesta desde la modernidad (Valdés, s.f.).

Sucede también que el protagonismo que han ganado los indígenas urbanos ha generado ciertas suspicacias de parte de diversas organizaciones indígenas más radicales ligadas a los movimientos de recuperación de tierras. Estas lo ven como una estrategia de parte del Estado para dividir al pueblo mapuche, con la idea de que los primeros posterguen a los 
movimientos de reivindicación de tierras y por la autonomía territorial a un lugar secundario. Sin embargo, hemos observado que, incluso desde la ciudad, el asunto del territorio sigue siendo el discurso que se posiciona con más fuerza. La imagen del mapuche sigue ligada a la comunidad del sur y, por lo tanto, desde ahí se construyen sus referentes políticos. La imagen del weichafe (guerrero) y el werken (mensajero) del sur han cobrado presencia en los discursos políticos, incluso entre los dirigentes urbanos.

En este sentido, la agrupación en torno a objetivos culturales —como la realización de prácticas de carácter ancestral — y demandas materiales - como lugares de reunión, vivienda, trabajo y educación con pertinencia cultural-, ha sido enmarcada en un contexto de mucha reflexión y accionar político, donde una de sus expresiones es, precisamente, el surgimiento de oficinas de asuntos indígenas en Santiago. Sin embargo, este proceso no ha sido lo suficientemente documentado y analizado como otra expresión posible del movimiento mapuche contemporáneo.

\section{Transición a la democracia, Ley Indígena y políticas públicas}

Durante los últimos años de la dictadura de Pinochet las organizaciones indígenas comenzaron a posicionar un fuerte discurso que introdujo la variable étnica y de derechos a la serie de demandas que en esa década se reclamaban a lo largo de todo el país. Tales demandas fueron recogidas por la oposición al régimen, conformada por la Concertación de Partidos por la Democracia y su candidato Patricio Aylwin, quienes el 12 de octubre de 1989 presentaron una Propuesta Programática para los Pueblos Indígenas que quedó plasmada en un acta conocida como el Acuerdo de Nueva Imperial, realizado en diciembre del mismo año. Este acuerdo sentó un precedente de diálogo entre quienes luego constituirían el poder ejecutivo y los pueblos indígenas, mismo que, en términos generales, guiaría el enfoque de la política indígena de los gobiernos de la transición democrática. Básicamente, las demandas establecidas en tal acuerdo estribaban en el reconocimiento constitucional de los pueblos indígenas, la creación de una corporación de desarrollo indígena y de un fondo de etnodesarrollo, y la creación de una comisión especial de pueblos indígenas para la implementación de la corporación, la elaboración de una ley específica y la ratificación del Convenio 169 de la Organización Internacional del Trabajo (OIT).

Al resultar electo, Patricio Aylwin convocó en 1990 a la Comisión Especial de Pueblos Indígenas (CEPI), la que elaboró un anteproyecto sobre la materia. Con base en este, el ejecutivo mandó tres proyectos de ley al Congreso: uno que incluía las demandas, otro para la reforma y reconocimiento constitucional de los pueblos indígenas y un tercero para la ratificación del Convenio 169 de la OIT. Solo fue aprobado el primero ${ }^{7}$ y en el año 1993 se decretó, con modificaciones, la Ley 19.253 sobre Protección, Fomento y Desarrollo de los Indígenas, conocida como la Ley Indígena.

Algunos de los artículos de esta ley hacen referencia a:

- La creación de la Corporación Nacional de Desarrollo Indígena (CONADI), “como un servicio público, funcionalmente descentralizado, dotado de personalidad jurídica y

${ }^{7}$ El Convenio 169 recién fue ratificado el año 2008, mientras que aún no se concreta el reconocimiento constitucional 
patrimonio propio, sometido a la supervigilancia del Ministerio de Planificación y Cooperación"8 (art. 38). Que tiene su sede en Temuco y cuenta desde el año 1996 con una Oficina de Asuntos Indígenas en Santiago.

- Las funciones de CONADI, organismo "encargado de promover, coordinar y ejecutar, en su caso, la acción del Estado a favor del desarrollo integral de las personas y comunidades indígenas, especialmente en lo económico, social y cultural y de impulsar su participación en la vida nacional"(art. 49).

- La calidad de indígena. Según el artículo 2 se considerarán indígenas a las personas de nacionalidad chilena "hijos de padre o madre indígena cualquiera sea la naturaleza de su filiación, inclusive la adoptiva; Se entenderá por hijos de padre o madre indígena a quienes desciendan de habitantes originarios de las tierras"; "siempre que posean a lo menos un apellido indígena" reconocido por CONADI; y "los que mantengan rasgos culturales de alguna etnia indígena entendiéndose por tales la práctica de formas de vida, costumbres o religión".

- Las asociaciones indígenas, las cuales según el artículo 36 se entienden como una "agrupación voluntaria y funcional integrada por, a lo menos, veinticinco indígenas que se constituyen en función de algún interés y objetivo común.

- La calidad de indígena urbano que, según el artículo 75, son quienes "se autoidentifiquen como indígenas y cuyo domicilio sea un área urbana del territorio nacional". Los indígenas urbanos "podrán formar Asociaciones Indígenas Urbanas o Migrantes" (art. 76). La CONADI "podrá impulsar y coordinar con los Ministerios, Municipios y oficinas gubernamentales planes y programas que tengan por objeto lograr mayores grados de bienestar para los indígenas urbanos" (MIDEPLAN, 1993).

Si bien la Ley Indígena de 1993, previo paso por el Parlamento, no alcanzó a recoger la totalidad de las demandas plasmadas en el Acuerdo de Nueva Imperial y luego se mostró débil en su implementación, resultó ser la primera vez que el Estado abordaba una política enfocada en los temas indígenas con carácter nacional (De la Maza, 2007; Campos, 2008), en la cual fue incluida explícitamente la población indígena en contexto urbano.

Dentro de las funciones de la CONADI se vislumbran, por tanto, puntos fundamentales que han promovido la participación de actores indígenas, tanto para la preservación y la reactivación de tradiciones culturales, como para la formulación de políticas y programas destinados a su desarrollo. Esto ha promovido la organización legal por parte de los actores indígenas que habitan las ciudades, pero también ha generado una competencia por los recursos, ya que los objetivos que la organización debe explicitar tienen que ver, generalmente, con la atribución de fondos para su desarrollo. Producto de ello, las asociaciones se han multiplicado, así como también sus demandas, las que se dirigen principalmente hacia los gobiernos locales. Por otro lado, los fondos de la CONADI han resultado insuficientes, pues se han enfocado, sobre todo durante los primeros años de funcionamiento, en la adquisición de tierras para las comunidades rurales del sur, postergando con ello las demandas de los habitantes de las ciudades.

El año 2000, durante el gobierno de Ricardo Lagos (2000-2006) y al alero del Ministerio de Planificación, se constituyó un Grupo de Trabajo para los Pueblos Indígenas que, en cincuenta días, recogió las demandas de los indígenas y las plasmó en un documento entregado al presidente, quien como respuesta redactó la "Carta del Presidente a los

\footnotetext{
${ }^{8}$ Ministerio que el año 2011 pasa a ser el Ministerio de Desarrollo Social.
} 
Pueblos Indígenas de Chile", en la que se decretaron 16 medidas a ejecutar por medio de un programa interministerial. Medidas que, entre otras, promovieron la creación de la Comisión Verdad Histórica y Nuevo Trato con los Pueblos Indígenas, que fue conformada por diversos colectivos representantes de los pueblos indígenas y el mundo académico y político del país (CVHYNT, 2008). Con base en el trabajo realizado por esta comisión se elaboró un informe que, si bien puede ser considerado de gran valor histórico, no ha tenido hasta la fecha un mayor impacto en la política nacional (De la Maza, 2007). Dentro de estos grupos de trabajo se encontraba uno conformado específicamente por indígenas urbanos, quienes entregaron los antecedentes sobre la situación de los indígenas migrantes en la ciudad, concretamente en la Región Metropolitana.

Durante el gobierno de Michelle Bachelet (2006-2010) se anunció una nueva política urbana, para la cual se conformó una comisión mixta con representantes de organizaciones indígenas y representantes del gobierno. Entre los meses de diciembre de 2006 y abril de 2007 se llevó a cabo la Consulta Nacional Indígena Urbana, a través de más de 20 encuentros locales y nacionales y con la participación de más de dos mil dirigentes, cuyo informe final presentó una propuesta participativa para una política para indígenas urbanos, que culminó con la creación del Programa Indígena Urbano (PIU) durante el gobierno de Sebastián Piñera (2010-2014).

El PIU tuvo como misión "promover el desarrollo integral de la población indígena urbana favoreciendo el acceso a oportunidades de emprendimiento, generación de capital social, espacios de participación, así como el rescate y valoración de su identidad en el contexto de una sociedad inclusiva y democrática" (www.conadi.gob.cl). Sus lineamientos estratégicos fueron: 1) fortalecer las formas de participación de los pueblos indígenas, en tanto actores de nuestra sociedad en el ámbito político y social; 2) reconocimiento y profundización de los derechos; 3) la Política Indígena Urbana; 4) la mujer indígena; y 5) educación y cultura.

Los derechos que este programa promovió fueron el derecho a la identidad, diversidad, participación y autogestión; y sus objetivos apuntaron a la reparación de la deuda histórica y a la construcción de un país multicultural y pluriétnico. Para esto, el PIU se enfocó en generar programas y proyectos orientados a indígenas urbanos que mejoren su calidad de vida y su acceso a la protección social, salud, medio ambiente, vivienda e ingresos económicos, con la inclusión de elementos de identidad y culturales propios.

A nivel local, específicamente en la Región Metropolitana, los encargados de llevar a cabo el PIU fueron la CONADI, a través de su sede en Santiago; los municipios, a través de las OAIs y la Mesa de Planificación Local Intercultural; y las organizaciones indígenas locales. Esto le otorgó un papel clave a las OAIs en la integración de tales proyectos interculturales a la estrategia de desarrollo comunal y la incorporación de la variable indígena en el plan de desarrollo comunal (PLADECO) de cada comuna.

\section{Oficinas de Asuntos Indígenas. La primera experiencia en la comuna de La Pintana.}

Debido a la conjunción entre la promulgación de la Ley Indígena, los procesos de reactivación cultural descritos previamente y el porcentaje de población mapuche que 
reside en la Región Metropolitana, hasta el año 2015 se habían registrado en CONADI ${ }^{9}$ doscientas catorce asociaciones indígenas. De estas, ciento noventa y siete asociaciones son mapuche, dentro de las cuales solo el $36.5 \%$ se encontraba vigente y realizando actividades, principalmente medicinales, educativas y culturales, mientras que el resto, solamente inscritas, sin actividad registrada.

Algunas de estas asociaciones, en conjunto con diversas organizaciones indígenas municipales, han optado por acercase a los gobiernos locales y posicionar sus demandas, ya sea a través de comités, de la Dirección de Desarrollo Comunitario (DIDECO) o negociando directamente con los alcaldes. Este proceso de participación y negociación con los municipios ha motivado una respuesta por parte de tales instituciones que se han materializado en la creación de una OAI. En un primer momento, estos espacios, que en su mayoría han sido gestionados y administrados por los mismos actores indígenas habitantes de las comunas, han tenido como encargados a representantes indígenas validados por la población indígena de la comuna, independiente, muchas veces, de su trayectoria política anterior o formación profesional.

Actualmente existen en la Región Metropolitana un total de 52 comunas, de estas, como ya fue señalado, las que cuentan actualmente con una oficina dedicada a los asuntos indígenas son $27^{10}$, lo que equivale al $51,92 \%$ del total. Estas comunas son Cerrillos, Cerro Navia, El Bosque, Estación Central, Huechuraba, Isla de Maipo, La Cisterna, La Florida, La Granja, La Pintana, Lampa, Lo Barnechea, Lo Espejo, Lo Prado, Maipú, Padre Hurtado, Pedro Aguirre Cerda, Peñalolén, Pudahuel, Quilicura, Quinta Normal, Recoleta, San Bernardo, San Joaquín, San Miguel, San Ramón y Santiago Centro (ver cuadro 1).

\section{Municipalidades de la Región Metropolitana con OAI}

\begin{tabular}{|l|l|l|}
\hline $\mathbf{N}^{\mathbf{0}}$ & Nombre Oficina & Comuna \\
\hline $\mathbf{1}$ & Oficina de Asuntos Indígenas & Cerrillos \\
\hline $\mathbf{2}$ & Oficina de Asuntos Indígenas & Cerro Navia \\
\hline $\mathbf{3}$ & $\begin{array}{l}\text { Programa de Pueblos } \\
\text { Originarios }\end{array}$ & EI Bosque \\
\hline $\mathbf{4}$ & Oficina de Asuntos Indígenas & Estación Central \\
\hline $\mathbf{5}$ & Oficina de Asuntos Indígenas & Huechuraba \\
\hline $\mathbf{6}$ & Oficina de Asuntos Indígenas & Isla de Maipo \\
\hline $\mathbf{7}$ & Oficina de Asuntos Indígenas & La Cisterna \\
\hline $\mathbf{8}$ & Oficina de Asuntos Indígenas & La Florida \\
\hline $\mathbf{9}$ & Oficina de Asuntos Indígenas & La Granja \\
\hline $\mathbf{1 0}$ & Oficina de Asuntos Indígenas & La Pintana \\
\hline $\mathbf{1 1}$ & $\begin{array}{l}\text { Oficina de Pueblos } \\
\text { Originarios }\end{array}$ & Lampa \\
\hline $\mathbf{1 2}$ & Oficina de Asuntos Indígenas & Lo Barnechea \\
\hline $\mathbf{1 3}$ & Oficina de Asuntos Indígenas & Lo Espejo \\
\hline $\mathbf{1 4}$ & Oficina de Asuntos Indígenas & Lo Prado \\
\hline
\end{tabular}

\footnotetext{
${ }^{9}$ Información obtenida a través de solicitud directa en CONADI de registro de asociaciones indígenas.

${ }^{10}$ Más el Departamento de diversidad y no discriminación de la Municipalidad de Providencia que cuenta con un encargado en asuntos indígenas.
} 


\begin{tabular}{|l|l|l|}
\hline $\mathbf{1 5}$ & Oficina de Asuntos Indígenas & Maipú \\
\hline $\mathbf{1 6}$ & Oficina de Asuntos Indígenas & Padre Hurtado \\
\hline $\mathbf{1 7}$ & Oficina de Asuntos Indígenas & Pedro Aguirre Cerda \\
\hline $\mathbf{1 8}$ & $\begin{array}{l}\text { Oficina de Pueblos } \\
\text { Originarios }\end{array}$ & Peñalolén \\
\hline $\mathbf{1 9}$ & Oficina de Asuntos Indígenas & Pudahuel \\
\hline $\mathbf{2 0}$ & $\begin{array}{l}\text { Oficina de Pueblos } \\
\text { Originarios }\end{array}$ & Quilicura \\
\hline $\mathbf{2 1}$ & Oficina de Asuntos Indígenas & Quinta Normal \\
\hline $\mathbf{2 2}$ & Oficina de Asuntos Indígenas & Recoleta \\
\hline $\mathbf{2 3}$ & Oficina de Asuntos Indígenas & San Bernardo \\
\hline $\mathbf{2 4}$ & Oficina de Asuntos Indígenas & San Joaquín \\
\hline $\mathbf{2 5}$ & Oficina de Asuntos Indígenas & San Miguel \\
\hline $\mathbf{2 6}$ & Oficina de Asuntos Indígenas & San Ramón \\
\hline $\mathbf{2 7}$ & Oficina de Asuntos Indígenas & Santiago \\
\hline
\end{tabular}

Cuadro 1. OAIs en la Región Metropolitana. Elaboración propia con información de la CONADI y consulta directa en las municipalidades.

La primera apertura de una oficina de asuntos indígenas se registra en la comuna de La Pintana en el año 1996, mismo año en que la CONADI instala su sede en Santiago. Creemos que este caso refleja, en buena forma, los caminos, tensiones, alcances y limitaciones que caracterizan la gestión de las OAIs en la Región Metropolitana. Por ello, describiremos brevemente la historia de su conformación y posterior desarrollo.

La OAI de La Pintana surge, precisamente, como respuesta a las demandas de la población mapuche de la comuna - expresadas a través de una asociación indígena y de su dirigente - para conseguir un espacio pertinente para la realización de prácticas rituales y culturales de carácter ancestral a partir de un hecho en particular. Durante los primeros años de la década de 1990, diversos grupos de mapuche residentes en la ciudad lograron gestionar la facilitación de un terreno perteneciente a la congregación del Verbo Divino en la comuna de La Florida, donde durante varios años realizaron encuentros y ceremonias en torno a la cultura mapuche, convocando a personas de diferentes comunas. Sin embargo, en 1996 gran parte de este espacio fue solicitado de vuelta por la congregación y puesto a la venta ${ }^{11}$, lo que dejó de nuevo sin un lugar adecuado para la realización de reuniones y celebraciones a diversas agrupaciones que habían encontrado ahí el contexto idóneo para la recreación de sus tradiciones. Entre estas agrupaciones se encontraban las de la comuna de La Pintana. La necesidad de gestionar un nuevo lugar determinó que una de estas asociaciones decidiera dirigirse directamente al alcalde de ese entonces, Jaime Pavez ${ }^{12}$, para solicitarle que la municipalidad pudiera gestionar la implementación de un espacio ceremonial en un sitio fiscal. Tal demanda contó con una buen a acogida y de esta manera se construyó la primera ruka en la comuna, en un

\footnotetext{
${ }^{11}$ Gran parte de este terreno se vendió para la construcción de un centro comercial. Aunque la congregación sigue realizando encuentros con personas mapuche, estas actividades, con el tiempo, adoptaron un carácter cristiano y terminaron configurando una pastoral mapuche.

12 Jamie Pavez, militante del centroizquierdista Partido por la Democracia (PPD), fue alcalde La Pintana entre 1992 y 2016. Para el periodo 2016 y 2020 fue electo como concejal.
} 
principio con fines medicinales, permitiendo además la participación de un dirigente indígena en el espacio municipal (Carmona, 2014).

La gestión de este espacio a través del municipio tenía como objetivo conseguir recursos y apoyo institucional para la conformación de una oficina indígena de carácter autónomo, desde la cual poder administrar y organizar los temas y actividades relacionados con la población mapuche de la comuna. Esta expectativa fue abordada de manera parcial, pues el alcalde, si bien accedió a su conformación, inscribió a esta oficina dentro del aparato administrativo, haciéndola dependiente de DIDECO. Sin lineamientos claros, tanto por parte de las organizaciones como del municipio, y sin un presupuesto aprobado, fue decretada en 1996 y comenzó a funcionar en 1997. El primer encargado fue el presidente de la organización mapuche Inchiñ Mapu, José Painequeo, propuesto por el alcalde a causa de su trayectoria dirigencial y electo a partir de una terna propuesta por las mismas organizaciones de la comuna.

La OAI de La Pintana, aparte de ser la primera oficina de Santiago, es ejemplo de un trabajo construido en conjunto entre la municipalidad y la acción de los dirigentes. La ruka comenzó su funcionamiento como centro de salud, con financiamiento del Ministerio de Salud y, aunque ya no existe, dio origen a un proyecto más grande, ubicado al costado del campus Antumapu de la Universidad de Chile, en un terreno cedido en comodato a la organización Inchiñ Mapu, donde hasta hoy funciona uno de los centros ceremoniales de la comuna ${ }^{13}$. Este centro cuenta actualmente con dos rukas, la más antigua es administrada por la organización, y la otra por la OAI. Esta última cumple la función, por un lado, de acoger las actividades de carácter institucional organizadas por la oficina y, por el otro, alojar a otras organizaciones de la comuna que no cuentan con su propio espacio o ruka.

Según Painequeo, en un comienzo la oficina se constituyó prácticamente con él y una mesa. Durante más de diez años, este encargado logró progresivamente conseguir recursos para la gestión y la administración de los programas sociales con enfoque indígena. Bajo su conducción se establecieron los lineamientos de la oficina en el contexto municipal y se promovieron actividades culturales de difusión y celebración de tradiciones del pueblo mapuche. Sin embargo, el hecho de que el encargado perteneciera a una organización en particular desencadenó algunos conflictos y desconfianza por parte de otras organizaciones de la comuna, debido principalmente a la competencia por los recursos municipales y estatales. Si bien esta situación puede tener sus matices y no nos corresponde determinar si tales sospechas fueron infundadas o justificadas, podemos comprender que los intereses de las asociaciones terminaran por entrar en conflicto dada la competitividad que provoca el diseño de las políticas públicas y sus concursos, lo cual promovió un clima de tensión y la sensación de una administración parcial.

Otro factor que repercutió sobre la gestión del encargado fue el aumento de exigencias institucionales que progresivamente fueron sentando las nuevas directrices en los cargos municipales. Entre estas destaca la rendición de cuentas. Acostumbrados a funcionar de manera local y con contratos directos, los municipios tuvieron que empezar a rendir cuenta de cada uno de sus gastos, adquisiciones y contrataciones a la Contraloría, debiendo por tanto licitar la mayoría de sus necesidades y adquirir sus productos a través

\footnotetext{
${ }^{13}$ Hoy son tres los centros ceremoniales activos que cuentan con rukas en la comuna, además de otras dos más pequeñas, una en un jardín infantil, gracias al financiamento del PEIB, y otra en un consultorio a través del PESPI. Esto hace de La Pintana la comuna con más rukas de la Región Metropolitana.
} 
de ChileCompra, portal de la institución que administra el sistema de compras públicas de Chile y que funciona con un marco regulatorio único con proveedores autorizados. Esto, evidentemente, ha sumado nuevas exigencias al perfil de los funcionarios municipales, entre ellos al encargado de la OAI, y ha determinado que los gobiernos locales demanden profesionales cada vez más especializados por sobre dirigentes sociales.

La confluencia de estos dos factores - la disputa por los recursos entre las organizaciones de la comuna y la profesionalización de los funcionarios municipales-, determinó que el alcalde tomara la decisión de reemplazar al encargado por un profesional con competencias para sistematizar la información y ajustarse a los procedimientos de administración y rendición de recursos. Para esto se optó por una trabajadora social no indígena que trabajaba en otra dependencia municipal. Según el alcalde, su elección se basó en el hecho de que esta profesional administraba la Beca Indígena (BI) ${ }^{14}$, una subvención dirigida a la población indígena que cursa estudios primarios, secundarios y universitarios, por lo que contaba con conocimiento de la población indígena de la comuna y ya estaba levemente familiarizada con la oferta pública para personas indígenas. De todos modos, el primer funcionario no fue despedido, sino que fue designado como asesor cultural y quedó a cargo de la organización de las actividades culturales.

Aunque el presupuesto de la OAI fue progresivamente incrementado, este no superó los 50.000 USD al año, es decir, bastante restringido, sobre todo si consideramos que en la actualidad la OAI cuenta con tres funcionarios, además de los dos ya mencionados, una funcionaria mapuche que desempeña labores de apoyo técnico.

Bajo la lógica municipal, la OAI opera como un programa, ya que su presupuesto se aprueba y renueva año a año. Sin embargo, los funcionarios prefieren nombrarla como oficina pues consideran que el apelativo de programa remite a una instancia de carácter asistencialista, lo cual va en contra del principal objetivo de la OAI: fomentar el fortalecimiento de los dirigentes, preservando al mismo tiempo la cultura de carácter ancestral. Para lograrlo, en todo caso, no se descarta el trabajo en conjunto con programas sociales, ya que estos son considerados como una vía para acceder a una red de recursos y con ello a la materialización de determinadas demandas.

El rol de la OAI es la coordinación de programas, en tanto instancia facilitadora y mediadora entre las organizaciones y el Estado. El eje de su gestión, alineado a los objetivos de la administración municipal, ha sido la integración de las asociaciones, entre sí y con la comunidad en general. Para esto ha logrado instalar una mesa de coordinación que funciona una vez al mes, en la que la oficina y las asociaciones realizan una planificación y evalúan las actividades comunitarias, como el año nuevo mapuche y el nguillatun, y la conducción de la oficina. La OAI de La Pintana trabaja con la mayoría de las organizaciones de la comuna ${ }^{15}$, todas mapuche, las que se acercan fundamentalmente por tres motivos: i) tramitación y obtención de la Beca Indígena; ii) apoyo y orientación

\footnotetext{
${ }^{14}$ Esta beca es entregada en conjunto por la Junta Nacional de Auxilio Escolar y Becas (JUNAEB), dependiente del Ministerio de Educación, y la CONADI. Consiste en un aporte monetario de libre disposición para estudiantes de ascendencia indígena con situación socioeconómica vulnerable y buen rendimiento académico, con el fin de facilitar su acceso al sistema educativo, Recuperado en: www.junaeb.cl.

${ }^{15}$ Las que fluctúan entre 10 y 14, dependiendo del año y la renovación de su personalidad jurídica. Actualmente todas son mapuche, sin embargo, durante algunos años hubo una diaguita, que por falta de integrantes no pudo continuar.
} 
para conformar o ingresar a una organización indígena; e iii) información sobre beneficios en temas de vivienda.

Los funcionarios de la OAI son concientes sobre la importancia que ha significado este espacio para la comuna, pues ha facilitado las vías para que el municipio aplique una política con mayor pertinencia y eficacia, lo cual ha propiciado el reconocimiento de la situación de las personas mapuche y de la importancia de su cultura. La coordinación y el apoyo a las organizaciones a través de la consolidación de una mesa de trabajo — que en sí misma se considera un logro-, ha promovido y fortalecido la asociatividad, facilitando la reagrupación de sus integrantes en la ciudad y aumentado su autoestima. De esta manera, la Oficina de Asuntos Indígenas de La Pintana se ha constituido como un referente en materia de política municipal indígena, tanto a nivel regional como nacional, reflejo de lo anterior es la replicación de este modelo en otros 26 municipios de la región y otros a nivel nacional. Mas, este espacio no está libre de limitantes y vulnerabilidad: su presupuesto es muy reducido y, debido a que las OAIs aún no están normadas, pues la Ley Orgánica de Municipalidades no hace mención sobre la necesidad de estos espacios ni siquiera en lugares de gran población indígena, su continuidad año a año está sujeta a la voluntad política del alcalde. A pesar de lo anterior, la OAI ha logrado posicionarse y fortalecerse en el tiempo, permitiendo una canalización de recursos y un espacio de participación permanente que anteriormente no existía, lo que a su vez repercute en el, aún incipiente, carácter intercultural del municipio y la percepción de la comunidad local.

\section{Conclusiones}

Debido a influencias de orden tanto internacional como nacional, durante las últimas décadas el Estado chileno se ha visto emplazado a repensar la relación con los pueblos indígenas, y en especial con el pueblo mapuche, respondiendo a través de una institucionalidad y políticas públicas diferenciadas que han repercutido en los procesos locales y en la emergencia de nuevos actores políticos (Espinoza, 2011).

Al observar el surgimiento y la evolución de las OAIs, se observa que la mayoría han surgido gracias a la iniciativa de los propios habitantes indígenas de las comunas, quienes al percibir en los gobiernos locales una posibilidad de participación política e inclusión de sus demandas, han gestionado los espacios y recursos inscritos en los márgenes de la estructura estatal y, con ello, redireccionado, aunque sea mínimamente, la agenda y el presupuesto del municipio.

La emergencia y la evolución de dirigentes y de las OAIs guarda directa relación con la reactivación de la cultura que ha tenido lugar durante las últimas décadas en la Región Metropolitana, ya que muchas veces las negociaciones apuntan a conseguir los espacios y los recursos necesarios para la realización de prácticas de carácter ancestral y la construcción de lugares acordes. Esta relación entre identidad cultural y política trajo consigo una reconfiguración y politización de la cultura, producto de la adopción de los mecanismos políticos institucionalizados por el Estado. 
Empujados por una búsqueda de reconocimiento y de un espacio de participación, los indígenas que han gestionado y administrado una OAI le han dado paralelamente forma a espacios exclusivos para la ejecución de las políticas públicas del Estado enfocadas al mundo indígena, apropiándoselas y resignificándolas. Tal apropiación implica una postura por parte del funcionario indígena, quien al vincularse con el Estado dinamiza las lógicas de las asociaciones, promoviendo "nuevos alineamientos y alianzas entre los distintos actores en la lucha por el control de recursos o en la búsqueda de legitimidad política de los liderazgos frente a la comunidad, el Estado, los partidos políticos, o ambos" (Bello, 2004: 66).

El surgimiento de la OAI en la comuna de La Pintana marca, por lo tanto, un hito dentro de los procesos etnopolíticos de la Región Metropolitana, y debido a esto se ha posicionado como un modelo a seguir por diversos municipios del país. Aunque no exista una legislación respecto a su funcionamiento, las OAIs se han multiplicado, especialmente en el transcurso de los últimos diez años. Esto ilustra la capacidad de agencia de los propios actores locales para gestionar recursos, reorientar la política en espacios institucionales y posicionar la identidad étnica como centro de su accionar político. Pero también, la identidad mapuche a la que se adscribe ha debido reconstituirse en función de la institucionalidad, configurando y desviando a la vez a esta última hacia las demandas propias de esta nueva contextualización.

Este proceso, no obstante, aún no puede ser completamente evaluado en cuanto es reciente y también se está modificando, con avances y retrocesos. Resulta, sin embargo, una instancia que permite reflexionar sobre las variaciones que configuran tanto al mundo mapuche que vive en la Región Metropolitana, como la relación del Estado con los pueblos indígenas, aminorando, por ejemplo los niveles de discriminación existentes en muchas comunas. Y si bien se puede afirmar que la distribución del poder sigue siendo enormemente desigual y que las condiciones en las que estos espacios se desarrollan siguen siendo profundamente vulnerables, del mismo modo podemos subrayar que el poder deja siempre intersticios, espacios grises donde los menos poderosos pueden buscar posicionar sus demandas. Espacios de fuerza centrípeta, puede ser, pero que de todos modos arrojan a los sujetos a nuevos lugares, más cerca del centro, pero también más lejos de la exclusión.

\section{BIBLIOGRAFÍA}

ANCÁN, J. (1993) "Rostros y voces tras las máscaras y los enmascaramientos: Los Mapuche urbanos. Kallfü trayenko. El color en la cultura mapuche" - Nütram- Año IX, No33- Santiago, Chile.

ANIÑIR, D. (2009) Mapurbe -Venganza a raíz - Pehuén editores- Santiago, Chile.

ANTILEO, E. (2008) Reflexiones de organizaciones Mapuche en torno a la problemática de la urbanidad. Memoria para optar al título de Antropólogo Social, Universidad de Chile, Recuperado en:

http://www.tesis.uchile.cl/tesis/uchile/2008/antileo_e/html/index-frames.html 
ARAVENA, A. (2003) "Los mapuche-warriaches; procesos migratorios contemporáneos e identidad mapuche urbana" (versión corregida y aumentada) - América IndígenaVol.LIX No 4 Oct.-Dic., pp. 162-188- México.

BARTOLOMÉ, M. (2006) Procesos Interculturales: antropología política del pluralismo cultural en América Latina- Siglo XXI - México.

BELLO, A. (2002) "Migración, identidad y comunidad mapuche en chile: entre utopismos y realidades"- Asuntos Indígenas- No 3-4, (2002)- IWGIA - Copenhague, Dinamarca.

(2004) Etnicidad y ciudadanía en América Latina- Naciones Unidas- Santiago.

BENGOA, J. (2000) La emergencia indígena en América Latina- Fondo de Cultura Económica- Santiago de Chile.

BOCCARA, G. (2000) “Antropología diacrónica, Dinámicas culturales, procesos históricos y poder político"- en Lógica mestiza en América- Boccara, Guillaume y Galindo, Sylvia (eds.) -Instituto de estudios indígenas, Universidad de la FronteraTemuco, Chile.

CAMPOS, L. (2002) "La problemáticas indígena en Chile. De las políticas indigenistas a la autonomía cultural" - Revista Academia- No 7- Universidad Academia de Humanismo Cristiano- Santiago, Chile (Págs. 39-58).

CARMONA, R. (2014) Mapuche urbanos y gobierno local. Etnografía de la Oficina de Asuntos Indígenas comuna de La Pintana. Tesis para optar al grado de Magíster en Antropología, Universidad Academia de Humanismo Cristiano: Santiago.

(2015). "Rukas en la ciudad. Cultura y participación política mapuche"- Antropologías del Sur- $N^{o}$ 4- Universidad Academia de Humanismo Cristiano- Santiago, Chile (págs. 67-87).

COMISIÓN DE LA VERDAD HISTÓRICA Y NUEVO TRATO (CVHYNT). (2008). Informe de la Comisión Verdad Histórica y Nuevo Trato con los Pueblos Indígenas. Gobierno de Chile- Pehuén Editores- Santiago, Chile.

CUMINAO, C. Y ABARCA, G. (2002) "Diferentes visiones de autores acerca de la migración y de la identidad de los mapuche de Santiago de Chile". Documento preparado por Geraldine Abarca Carimán y Clorinda Cuminao Rojo por encargo del Grupo de Trabajo Indígenas Urbanos, de la Comisión de Verdad Histórica y Nuevo Trato.

DE LA MAZA, F. (2007) Política social para indígenas. Un análisis desde la perspectiva de la construcción del Estado y la interacción local, Panguipulli (1990-2005), Chile. Tesis de Doctorado en Antropología- Ciesas, D.F- México. 
DEVALPO, A. (2008) Pacífica oposición de los mapuche chilenos, en: Historia y luchas del Pueblo Mapuche - Editorial Aún Creemos en los Sueños- Santiago, Chile.

ESPINOZA, C. (2011) Mapuches y Poder Local, Transformaciones en el escenario político de Tirúa (1992-200), Chile. Tesis de Doctorado en Antropología- Ciesas, D.FMéxico.

LLAITUL, H. Y ARRATE, J. (2012) Weichan conversaciones con un weychafe en la prisión política- Ceibo- Santiago, Chile.

MIDEPLAN (1993) Ley 19.253, Establece normas sobre protección, fomento y Desarrollo de los indígenas, y crea la corporación Nacional de desarrollo indígena. Recuperado en: :https://www.leychile.cl/N?i=30620\&f=2017-11-03\&p=

MIDESOC (2015) Encuesta de Caracterización Socioeconómica (CASEN)- Recuperado en: $\quad$ http://observatorio.ministeriodesarrollosocial.gob.cl/casenmultidimensional/casen/casen_2015.php ,Santiago, Chile.

VALDÉS, M. (s/f) El problema de lo urbano y lo rural- Recuperado en: http://www.mapunet.org/documentos/mapuches/urb_rur.htm 